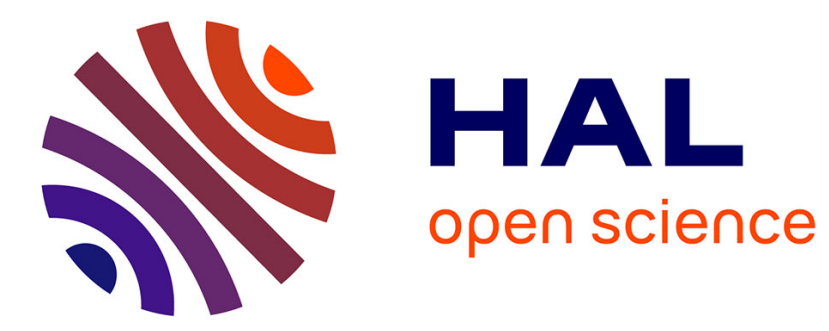

\title{
Des cadavres de militaires français morts hors du combat dans la Guerre de Crimée (1854-1856)
}

Benoît Pouget

\section{To cite this version:}

Benoît Pouget. Des cadavres de militaires français morts hors du combat dans la Guerre de Crimée (1854-1856). CORPS : Revue Interdisciplinaire, 2017. hal-01728360

\section{HAL Id: hal-01728360 https://hal.science/hal-01728360}

Submitted on 10 Mar 2018

HAL is a multi-disciplinary open access archive for the deposit and dissemination of scientific research documents, whether they are published or not. The documents may come from teaching and research institutions in France or abroad, or from public or private research centers.
L'archive ouverte pluridisciplinaire HAL, est destinée au dépôt et à la diffusion de documents scientifiques de niveau recherche, publiés ou non, émanant des établissements d'enseignement et de recherche français ou étrangers, des laboratoires publics ou privés. 


\title{
Des cadavres de militaires français morts hors du combat dans la Guerre de Crimée (1854-1856)
}

Benoît Pouget

\author{
Pour citer cet article : \\ Pouget Benoît, "Des cadavres de militaires français morts hors du combat dans la Guerre \\ de Crimée (1854-1856)», Corps, vol. 15, no. 2, 2017, p. 293-304.
}

«Et la vie de l'horloge d'ébène disparut avec celle du dernier de ces êtres joyeux. Et les flammes des trépieds
expièrent. Et les Ténèbres, et la Ruine, et la Mort rouge établirent sur toutes choses leur empire illimité. »
Edgar Allan Poe, Le masque de la mort rouge, traduction de Charles Baudelaire, Edgar Allan Poe, Le masque de la mort rouge, traduction de Charles Baudelaire,
1842.

La guerre de Crimée a été pour le corps expéditionnaire français un défi médical et sanitaire considérable (Gouttman, 2003 ; Lemaire, 2006 ; Gabriel, 2013). Le premier constat qui s'impose est quantitatif : le bilan des pertes est colossal. Le médecin-général Chenu dénombre 95615 tués, morts ou disparus sur un effectif de 309268 hommes de l'armée de terre et de mer envoyés sur le théâtre des opérations entre le $1^{\mathrm{er}}$ avril 1854 et le 6 juillet $1856^{1}$. C'est donc près d'un tiers du corps expéditionnaire qui succombe sur place ou après une évacuation. Toujours d'après Chenu, «seulement »10240 soldats Français tombent directement sous le feu de l'ennemi, qui provoque pour 39868 d'entre eux, des blessures requérant une prise en charge médicale. Le second constat marquant à propos des pertes est qualitatif autant que quantitatif : «les maladies tuent plus que les combats » (Arzalier, 2010). Choléra, scorbut, typhus, fièvres diverses sont en cause dans près de $90 \%$ des décès survenus dans le corps expéditionnaire. Dans un conflit qui peut être considéré comme l'un des « réels premiers exemples de guerre moderne », et au cours duquel de nombreuses innovations en matière d'armement furent éprouvées concernant notamment l'artillerie - 150 millions de cartouches et 5 millions de bombes et d'obus de divers calibre furent échangé par les belligérants des deux camps -, c'est finalement l'hiver et les grandes épidémies qui constituèrent le plus grand challenge à l'intégrité de la santé des soldats français (Figes, 2010 ; Holden Reid, 2001).

Au-delà du vertige des bilans, des enjeux du dénombrement et de la statistique sur lesquels l'historiographie récente s'est interrogée (Fredj, 2010), et en deçà d'un travail sur les causes de la mortalité dans les corps expéditionnaires européens au XIXème siècle (Curtin, 1989), le présent article souhaite rendre compte à l'échelle individuelle de la mort des soldats français tombés «naturellement » en Crimée, «dans ce dernier cas, les morts au combat [...] sont simplement sorties d'observation » (Houdaille, 1976). Nous excluons également de notre étude les cas de morts volontaires ou liées à un homicide volontaire. L'essentiel de l'article repose sur l'examen des pièces formant les 78 rapports de décès établis par les différents intervenants (rapports médico-légaux établis par le médecin militaire , rapport de l'officier supérieur, rapport de la gendarmerie ...) et conservés sous la côte G 259 au service Historique de la Défense à Vincennes ${ }^{2}$. Ils concernent des soldats mobilisés sur l'amplitude du front de la campagne de Crimée et dont le décès est intervenu sans lien direct avec l'épreuve du feu. Ce corpus n'est bien évidemment en aucun cas représentatif quantitativement des grandes tendances des causes de décès identifiées par Chenu, ni même certainement de l'amplitude des douleurs physiques ressenties par chaque soldat français qui rendit son dernier souffle en Crimée. Première cause de mortalité, Le choléra n'y est d'ailleurs que marginalement représenté alors qu'il emporte 11196 hommes dans le corps expéditionnaire entre 1854 et $1856^{3}$. 
Ce corpus est en définitive bien plus une somme de cas particuliers qui, s'il faut les replacer dans un contexte plus large, nous permettent d'attester à l'échelle individuelle des épreuves physiques endurées avant ou au moment d'expirer. Leur cadavre devenant le testament essentiel des souffrances accumulées et des conditions du trépas. Ils sont les traces matérielles de la forte surmortalité qu'entraînait la vie militaire au XIXème siècle, outre-mer comme en France (Houdaille, 1976, Smallman-Raynor \& Cliff, 2004). En s'attachant à rendre compte, très partiellement, de ces masses soldatesques défuntes passées dans les silences de l'histoire, il s'agit bien de chercher à approcher à partir de leurs cadavres au plus près de l'expérience de la mort de ces hommes tombés isolément loin du champ d'honneur. Dans quelles circonstances la Parque les saisit-elle ? En quoi leurs dépouilles attestent-elles tout à la fois de la singularité de leurs destinées autant que de l'épreuve collective d'une campagne militaire engagée loin de la France ? De l'agonie à la mise en terre, cette invitation à suivre ces hommes, conduit finalement à les réintégrer aux grands récits de la geste militaire, eux qui quittèrent ce monde sans même effleurer la gloire d'Hector ou de Turenne.

Traces matérielles s'il en est, la découverte de leurs cadavres met en mouvement une procédure médico-légale ordinaire qui s'achève avec l'inhumation du défunt. Les dossiers individuels ainsi constitués permettent de saisir leurs agonies dans leurs intimités, ce jusqu'à l'ultime souffle. Et d'alors remonter le fil des évènements pour les accompagner dans leurs derniers instants qui les mettent corps et âmes à l'épreuve.

\section{LE MASQUE DE LA MORT}

Les découvertes des cadavres des militaires français morts en dehors du combat recouvrent des situations et des contextes hétérogènes. Pour partie d'entre eux, la mort a frappé sous la tente. Soudaine, ou intervenant à la suite d'une phase d'agonie, elle fige de soldat dans son éternité. C'est le cas du dénommé Bernard, fusilier au $9^{\text {ème }}$ de ligne, retrouvé le 23 septembre 1855 sans vie sous sa tente au camp devant Sébastopol. Le décès est rapidement constaté par le docteur Coste - médecin du régiment- qui pratique alors l'examen médico-légal requis par le code pénal depuis 1810 (Trémaille, 2010). Il décrit alors le cadavre d'un homme jeune (22-23 ans) dont le visage ne manifeste «aucune expression de souffrance ». Il détaille alors un masque apaisé de la mort : « les yeux sont fermés, les pupilles dilatées et immobiles [...] aucune écume ni aucune mucosité sanguinolente ne s'échappent de la bouche [alors que] les battements du cœur sont entièrement suspendus ». Les descriptions de cadavres exempts de stigmates de souffrances ne recouvrent cependant qu'une partie de la réalité de l'expérience sensible du trépas. Nombre de corps portent en effet le témoignage d'une mort intervenue dans la souffrance. C'est évidemment le cas des hommes emportés par le choléra. Enlevé par l'épidémie qui affecte Gallipoli en juillet 1854, le Général Carburccia succombe le 17 après « quelques heures de souffrances ». Lui aussi dévasté par un « choléra foudroyant », le soldat Gesta, fusilier au 27ème de ligne, expire alors qu'on le transportait de la tente à l'ambulance de son régiment dans le camp de Traktir le 23 décembre 1855. C'est l'abdomen noué de crampes très violentes, les yeux caves, la figure amaigrie et la voix éteinte qu'il quitte ce monde sur un brancard.

La mort survient donc pour nombre de ces hommes dans la précipitation, sinon l'urgence. Elle est parfois accompagnée de signes avant-coureurs qui, paradoxalement, semblent très souvent aux yeux des contemporains déconnectés de la cause finale de décès. Le fusilier Bernier, émargeant au $79^{\text {ème }}$ de ligne (positionné à Sébastopol), dont la santé était déjà altérée par des engelures aux pieds et une diarrhée installée depuis 4 jours, est ainsi emporté le 9 mars 1855 sous la tente à la suite d'une suffocation subite sans que le rapport médico-légal ne tisse de lien entre les affections. Le 14 février 1856 le soldat Dussault du $98^{\text {ème }}$ de ligne défaillit lui aussi en «quelques instants [emporté] par une congestion pulmonaire [...] à la surprise de tout le monde » alors qu'aucun indice ne laissait présumer d'un tel sort : vers $19 \mathrm{~h} 30$ il est pris de 
douleur; son avant-bras est pris de gonflement; ses veines sont «noircies par l'action de la maladie » qui touche aussi la poitrine «qui devient bleu»; A 20 heures « il est décédé ».

Dans un contexte de promiscuité évidente crée par les conditions même de la campagne militaire, l'agonie et le trépas des soldats sont très souvent des moments partagés avec des camarades et un corps médical mobilisés pour les sauver. Le fusilier Pasquier du 64ème de ligne, manifestement affaibli par une fièvre, rend l'âme le 17 mars 1856 dans les bras de ses camarades que son « râlement prolongé » et sa «figure décomposée » avait tiré de leur sommeil. La fraternité des armes s'impose donc dans tous les moments de la vie militaire et pas seulement au feu. Premiers témoins du malaise, les camarades apportent souvent les premiers secours. Ceux du fusilier Dubois « s'empressent de lui donner des soins et ont appelé le médecin » alors qu'il est victime d'un anévrisme le 14 décembre 1855 sous sa tente dans le camp d'Inkerman. Si les premiers secours sont administrés par les camarades, le médecin prend aussi rapidement que possible le relais. Le fusilier Bernard, dont le corps inerte est transporté «immédiatement sous une tente d'infirmerie » le 23 septembre 1855, bénéficie de « la mise en œuvre de moyens énergiques pour le réanimer » : « ammoniaque liquide sous les narines, insufflation artificielle, compressions sur les parois de la poitrine » appliquées pendant près de 30 minutes n'arrivent pas à la ramener à la vie. Le fusilier du $27^{\text {ème }}$ de ligne Guhur reçoit les mêmes soins tandis qu'il agonise le 14 février 1856 : «il rend [alors] par la bouche un liquide noirâtre alors que l'on continue les pressions sur le thorax pour en favoriser l'écoulement ». Il est aisé d'imaginer dans de telles circonstances la dimension impressionnante de ces scènes d'agonies pour les témoins. Le Général Fay évoque à propos des ravages du choléra dans ses souvenirs de la Guerre de Crimée, l'impuissance des hommes qui «voyant leurs camarades tomber sous les coups invisible du fléau, les plus vaillants ne peuvent se défendre du découragement; ils tremblent de mourir ainsi, obscurément, loin du champ de bataille, où la mort frappe en face, et où ses victimes ne tombent pas du moins sans gloire» ${ }^{4}$.

Sous la tente, comme en terrain découvert, la mort reste le plus souvent une épreuve personnelle, solitaire. Elle intervient à l'abri des regards. Le cadavre du fusilier du $25^{\text {ème }}$ de ligne Jacoberger, retrouvé sur la grève de la baie de la Streletzka, en porte témoignage. Le médecin constate sur son corps la présence d'ecchymoses sur la face et le tronc alors que «l'épiderme se détachait en divers points ». Lorsque la mort survient sans témoin, le cadavre est en général découvert à distance du moment du décès. Il peut certainement en figer les circonstances. Le 30 juillet 1855, le médecin est ainsi appelé à 2 heures du matin afin de constater le décès du chasseur Dufaure du $10^{\text {ème }}$ Bataillon. Son corps a été retrouvé près du monastère Saint George. Un premier examen permet d'établir que la mort remonte à plusieurs heures. «Trois plaies étroites et profondes dans la région orbito-frontale droite » sont décrites dans son rapport. Elles « intéressent toute l'épaisseur des parois osseuses crâniennes ». Le 3 novembre 1855 la mort du caporal Blevanas des suites d' " une asphyxie par immersion » est, elle encore, intervenue dans la solitude des eaux de la baie de la Streletzka. C'est à moitié « plongé dans l'eau » que son corps fut retrouvé dans la matinée par des blanchisseurs sur la rive gauche de la baie. Transporté sur la rive droite, le médecin examine le cadavre d'un homme « couvert d'habits de petite tenue », en position de « décubitus dorsal avec une légère inclinaison à droite ». Si aucun signe de lutte n'est visible, la rigidité cadavérique « commençait à envahir » des articulations à demifléchies. Le cadavre du soldat Blevanas présentait toutes les caractéristiques médico-légales d'une noyade : sa «face bouffie [était] parsemée de marbrures livides » alors que « des liquides abondants s'écoulent par la bouche » et que « l'intervalle entre les ongles et la pulpe des doigts est remplis de sable ». Le corps est intact de toute autre plaie à l'exception d' " une cicatrice encore non consolidée présente à l'avant-bras droit causé par un impact de balle » reçue le 8 septembre 1855. La mer ou les lacs pouvaient d'ailleurs ne rendre les corps qu'après un séjour prolongé dans leurs eaux. Disparu à $25 \mathrm{~m}$ du rivage dans le lac de la Tchernaïa près du camp de Traktir le 19 mars 1855 sous les yeux de camarades alors qu'il voulait récupérer un canard 
blessé, le corps du soldat Gouéré du $30^{\text {ème }}$ de ligne n'émergea des eaux que le 15 avril après un mois d'immersion. C'est un cadavre présentant d'importantes lividités cadavériques « à la face surtout et aux membres » qui est alors examiné par le médecin du régiment. Il rend compte d'une « augmentation considérable du volume due à une putréfaction avancée » qui selon lui « a déterminé l'ascension du corps à la surface ».

\section{Translation DU MOURANT, GeSTION ET DESTINATION DE SA DEPOUILLE}

Ces soldats mourants et/ou leurs dépouilles sont dans tous les cas concernés par des dynamiques analogues de manutention. Dans l'urgence pour les vivants ou plus paisiblement pour les morts, l'infirmerie de l'ambulance du régiment ou l'hôpital de campagne sont leur destination finale. Lorsqu'ils sont considérés comme transportables, par le médecin ou par leurs camarades, les hommes sont donc dirigés vers l'espace de soins le plus proche. Ce moment de translation pouvant par ailleurs être l'occasion du décès. Souffrant d'une perforation de l'intestin causé par une dysenterie, le fusilier Primat est conduit à l'ambulance du camp de Sébastopol « vers trois heures du soir » le 22 février 1855. Appuyé sur le bras de deux camarades, il y est déposé sans vie. Pareille infortune arrive au fusilier Noirot du $98^{\text {ème }}$ de ligne le 8 décembre 1855. Effectuant des séjours réguliers à l'infirmerie du camp de Sébastopol depuis le début du mois d'octobre, il est emporté par une pleuropneumonie alors qu'il venait d'entrer «à l'ambulance de la $3^{\text {ème }}$ division du $1^{\text {er }}$ corps d'armée ». La même urgence préside au transport du fusilier Barbier (lui aussi du $98^{\text {ème }}$ de ligne) qui expire «à l'ambulance d'un autre régiment » où il avait été accueilli alors qu'il venait d'être victime d'une congestion cérébrale au retour d'une corvée à Sébastopol.

Lorsque la mort intervient ailleurs que dans les murs ou sous les tentes du service de santé des armées, c'est «à titre de dépôt » qu'est reçu le cadavre. Autant que la documentation l'étaye, tous les cadavres sont donc dirigés vers cette dernière étape avant l'inhumation. Retrouvé noyé dans une rivière le 15 juin 1854 , la dépouille du soldat Velche du $50^{\text {ème }}$ de ligne est reçue à l'hôpital d'Andrinople. A Constantinople, l'amphithéâtre de l'hôpital de DaoudPacha accueille le 18 novembre 1855 celle du dénommé Nollet. À Kamiesh, le corps sans vie du fusilier Barrot, mort aussi par noyade, est déposé le 31 juin 1855 à l'ambulance. La levée du corps, puis son acheminement du lieu de décès vers l'endroit qui fait fonction de morgue, s'effectue sur l'ordre d'un officier « remplissant les fonctions d'officier de l'État civil ». C'est ainsi que par exemple le 6 mars 1855 le sous-lieutenant Alix Charles François établit «après s'être déplacé sur le terrain » l'acte de décès du grenadier Delanoé mort d'un anévrisme. Il autorise ensuite « la levée du corps pour être transportée à l'ambulance de la deuxième division du $1^{\mathrm{er}}$ corps ». Cette corvée était généralement menée par un sous-officier et les hommes de troupes nécessaires. Reçus en tant que dépôt, les cadavres des soldats sont immobilisés le temps que soient remplies les formalités administratives (état civil, rapport médico-légal, rapport de gendarmerie, rapport du commandement). Lorsqu'il s'agit d'un personnel civil, comme dans le cas du cantonnier Croize mort à Varna des suites du choléra le 29 aout 1855, le consul de France est sollicité pour remplir ces fonctions d'État civil.

Dans l'écrasante majorité des cas documentés il est procédé à l'inhumation de la dépouille dans le cimetière attenant à l'ambulance de la division. Bazancourt ne dit autre chose lorsqu'il fait le constat qu'à Gallipoli « les tombes se creusaient silencieusement autour des camps » 5 . Décédé le 28 novembre 1855, «le cadavre du fusilier Jacques Eymat [...] a été inhumé au cimetière de la $1^{\text {ère }}$ division le même jour à 10 heures du matin ». Rien d'étonnant que de voir procédé avec une grande célérité à la mise en terre dans un contexte épidémique et sanitaire tendu (Lemaire, 2006). L'ensevelissement se faisait dans la terre où le corps recevait sa mesure de chaux. Le RP Damas se rappelle que «sur les hauteurs de Kamiesh, un four à chaux [brûlait] continuellement [...] surtout pour donner les moyens de consumer les cadavres dont la pourriture, sous l'ardeur du soleil, amènerait sans doute, l'infection et la mort» ${ }^{6}$. L'inhumation est 
finalement attestée par la délivrance d'un certificat qui en renseigne les conditions. Il est signé par l'officier supérieur du défunt. Mort du choléra, le fusilier Gesta est, comme son camarade

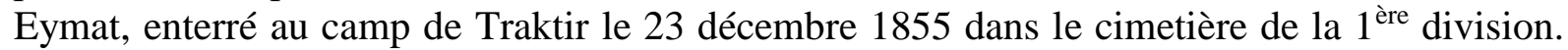
Quatre de ses frères d'armes ainsi que le lieutenant commandant la compagnie assistent à la mise en terre. Dans certaines circonstances, l'on pouvait procéder à l'inhumation in situ. Le cadavre du soldat Gouéré, découvert dans un état de décomposition avancé, est mis en terre très rapidement sur les bords du lac qui l'a englouti. On peut avancer sans risquer de se tromper comme hypothèse que son état ne permettait pas un transport jusqu'à l'ambulance et son cimetière.

\section{« LA MORT NE RESPECTE PERSONNE »}

Le capitaine Pruvost dans ses Souvenirs de la Campagne de Crimée l'exprime sans détour : «la mort ne respecte personne $»^{7}$. Plus encore, lorsqu'elle intervenait sans rapport avec le combat lui-même, elle n'était même pas « une consolation d'un juste sujet d'orgueil de se sentir frappé à son poste en accomplissant sa tâche et son devoir» ${ }^{8}$. Pas de mort en héros donc pour une majorité de soldats français en Crimée. Seulement leurs corps offerts en holocauste à un kaléidoscope de maux et de dangers. Chenu a procédé au classement minutieux des pathologies en cause afin d'en établir la nomenclature : fièvres, diarrhée, dysenterie, choléra, typhus, scorbut et maladies diverses ${ }^{9}$. S'appuyant sur le constat que «les soldats en guerre ne sont pas malades [et donc ne meurent pas] de la même manière que tout le monde », il fait état du caractère «spécial » de leurs maladies, qui conjuguent l'action « dans l'organisme d'un même sujet, de deux, quelquefois trois types d'altération morbide qui se disputent la vie du malade ${ }^{10}$. C'est donc sans grande surprise que ce constat de soldats atteints de plusieurs pathologies est documenté dans l'échantillon. Le cadavre du fusilier Guhur présentait ainsi à l'examen une «face pâle » ainsi que des lèvres, des gencives et une langue «bleuâtres » caractéristiques d'une affection scorbutique installée depuis longtemps. Cependant il succombe, avant que le scorbut n'achève son œuvre, d'une hémorragie interne procédant d'une « autre cause ». Décédé le 26 février 1855, le soldat Thiriet, qui meurt à la suite d'une apoplexie pulmonaire, était traité « depuis quelques jours à l'ambulance pour des problèmes de pied en mauvaise santé suite à une congélation ».

Les cas d' "asphyxie par le froid ou de congélation partielle des membres » étaient un en soi un défi sanitaire considérable. Le 5 janvier 1855 Chenu rapporte « un vent violent qui chasse une neige fine et rend le travail très pénible » avant d'ajouter que l'on constate des « congélations à tous les degrés ${ }^{11}$. Si la Crimée n'est pas l'Oural ou la Sibérie, les conditions atmosphériques qui s'imposent aux soldats ne les exposent pas moins. Dès le début de l'expédition, le 13 mai 1854, le conseil de santé des armées avait alerté les troupes sur la nécessité d' " être vêtu de manière à être à l'abri des refroidissements subits auxquels on est exposé en toute saison, à raison des brusques changements de température qui ont lieu fréquemment dans presque toutes les parties de ce pays $»^{12}$. Le péril est tel que le 4 janvier 1855 le Général en Chef Canrobert rappelle dans un ordre général « les règles à suivre en cas d'asphyxie par le froid $»^{13}$. Malgré ces multiples actions de prévention l'hiver tue en Crimée jusqu'à la fin de la campagne. Les 18 et 19 décembre 1855 par exemple, « des détachements considérables sont envoyés en corvée des camps [sur le plateau d'Inkerman] à Kamiesh pour rapporter du bois ; partis de grand matin [ils] ont à souffrir cruellement du froid $»^{14}$. Parmi ces hommes, les fusiliers Durocher et Leharivel. «Resté en arrière en arrière de la corvée au retour », Durocher tombe sur la route. Immédiatement recueilli par des « hommes du train », il est transporté dans le camp le plus proche où il rend l'âme. Comme pour son camarade Leharivel, c'est bien selon le médecin qui les examine l' " action vive et prolongé du froid [entrainant] une sidération sur système nerveux» qui est la cause de leur mort. Régulièrement, l'action du froid faisait son œuvre sans qu'aucun secours ne fût possible. Le cadavre du fusilier Beauplet est ainsi retrouvé 
le 16 décembre 1855 «enseveli sous la neige à quelques distances du port de Kamiesh ». Parti avec un camarade pour la corvée de vin, il se serait écarté du chemin, avant d'être pris par le froid alors qu'il se reposait près d'une maison en ruine. Son corps est apporté au médecin major du $98^{\text {ème }}$ de ligne qui ne peut que constater le décès «survenu depuis un peu plus de douze heures ». Les effets dévastateurs du froid sont par ailleurs amplifiés par les consommations excessives de boissons alcoolisées. Agent perturbateur de la discipline militaire, l'ivresse de soldats en campagne ne trouble pas [uniquement] «l'ordre public ${ }^{15}:$ « combien de marins et de soldats trouvés morts sur la neige pour avoir obéi au funeste penchant à la boisson ${ }^{16}$. Les autorités sont pleinement conscientes du danger mortel couru par les hommes. Canrobert alerte dans un ordre général sur «les dangers mortels de l'ivresse lorsque la température est froide [...] l'abus de liqueurs alcooliques amenant très vite l'asphyxie et la mort ${ }^{17}$. Parti lui aussi le 18 décembre 1855 pour la corvée de bois, le fusilier Langlois ( $79^{\text {ème }}$ de ligne) est incapable de retrouver son chemin alors qu' «il avait fait usage de liqueurs alcooliques ». Recueilli lui encore par des soldats du train, il est conduit chez le médecin où il reçoit des soins avant de retourner dans sa tente. Il est retrouvé mort le lendemain. L'ivresse était également en cause dans nombre d'accident. C'est à l'abus de boisson qu'est attribuée la chute du voltigeur Nollet dont le cadavre est retrouvé à l'intérieur d'un four à chaux "d'une profondeur de 10 à 12 mètres » au nord de l'hôpital de Daoud Pacha. La chute dans les eaux de la baie de la Strelitzka du caporal Blevanas est elle aussi imputée à une consommation d'alcool «outre mesure dans la journée ».

Rivières, fleuves, bord de mer ou de lacs, voici autant de lieu dangereux pour des hommes qui étaient loin de savoir universellement nager. «Forme principale de la mortalité violente aux époques anciennes », pour Jean-Claude Chesnais «la noyade occupe une très grande place surtout au $19^{\text {ème }}$ siècle, c'est de loin la cause prédominante » de décès par accident ; ses caractéristiques traditionnelles (chute dans un puits ou une rivière, enlisement dans un marécage, etc.) ne sont supplantés « par l'accident de baignade » qu'à la fin du siècle (Chesnais, 1976). Rien d'étonnant alors que de retrouver la noyade comme une cause de surmortalité accidentelle au cours de la campagne de Crimée. Trois exemples parmi de nombreux autres illustrent quelques-unes des conditions dans lesquelles pouvait survenir un décès pour cause d' «asphyxie par submersion ». D'abord, la traversée par voie de mer jusqu'à la Crimée était pleine de dangers pour des soldats «terriens » qui pouvaient se trouver dépourvus d'un pied marin. Embarqué à bord du Ripon, navire de guerre battant pavillon britannique, le sapeur conducteur (génie) Fournier est ainsi emporté en pleine mer le 21 janvier 1855 par une vague «sur le chemin des latrines ». Malgré la mise à l'eau du canot de sauvetage et de la bouée son corps est englouti au large du Cap Douro (Grèce). C'est en chutant dans les eaux de la baie de la Strelitszka le 31 juin 1855 que le fusilier Barrot succombe alors qu'il pratiquait la pêche au crabe avec un camarade. L'accident de baignade est documenté bien avant qu'il ne représente la première cause de noyade en France. Le 22 mai 1855 le fusilier Portalier se noie alors qu'il se baigne avec un camarade dans la baie de Kamiesh. L'année précédente, c'était dans une rivière près d'Andrinople que disparaissait dans des circonstances analogues le soldat Velche, et ce en dépit de l'intervention des «meilleurs plongeurs du régiment». Ces noyades sont à inscrire dans l'accidentologie qui à terre peuvent être attribués «à l'imprudence et à la maladresse des hommes ou à la rapidité de certaines manœuvre de force, pendant le déchargement et le transport du matériel ${ }^{18}$. En effet, «il en est beaucoup d'accidentelles [des morts] qui sont prévues et considérées avec raison comme événements de guerre, parce qu'elles sont inévitables et dépendent du déplacement ou de l'agglomération d'un grand nombre d'hommes ${ }^{19}$. Chenu distingue en particulier des accidents liés au déplacement et à l'entretien des animaux. Le 18 août 1854, le fusilier Cuvilier, muletier, meurt instantanément à Varna des suites d'une « blessure à la tête tellement grave » provoqué par une «hâte de voiture ». C'est bien un coup de corne «d'un bœuf de l'administration » qui tue le chasseur Dufaure dont les blessures ont déjà 
été évoquées. Dans l'inventaire qu'il donne des causes d'accident pour la population française au $19^{\text {ème }}$ siècle Jean-Claude Chesnais évoque les chutes, écrasement par des voitures (hippomobiles), incendies, éboulement de terrain ou de construction parmi les accidents les plus spectaculaires et les plus courants (Chesnais, 1976). Ils sont pour les soldats en Crimée autant d'accidents du travail qui les surprennent dans leurs tâches quotidiennes. Le 27 mai 1856 Le fusilier Bourdon ( $81^{\text {ème }}$ de ligne) est relevé sans connaissance après une chute de plus d'une vingtaine de mètre. L'accident s'est produit « alors qu'il était occupé à couper un arbre [...] près du pont de Creutzen à Alzou. Retrouvé en contrebas, «l'écume à la bouche et les yeux fixes », sa mort est «attribuée à l'intensité des phénomènes cérébraux » provoqué par l'impact avec le sol.

«La mort est mon métier » pourraient dire les soldats français embarqués dans l'aventure de l'expédition de Crimée. Cependant contrairement au personnage haïssable de Robert Merle qui la donne sans limites et sans remords, ces militaires la reçoivent en dehors du combat comme une fatalité imposée par les risques du métier. Leurs cadavres témoignent tout à la fois des circonstances et des causes de leurs décès. Ils rendent aussi compte des souffrances accumulées qui, liées directement ou non à leur trépas, sont autant de marques dans leur chair des dures conditions de la campagne. Au-delà de l'épreuve du feu, la résistance, et souvent la chance, des soldats sont intensément éprouvées par la vie quotidienne en arrière du front. Maladies et accidents les guettent sans répit. Les conditions mêmes de la campagne les obligent à se mouvoir sur des terrains tourmentés sur lesquels la concentration d'hommes favorise par la promiscuité qui en dérive la dynamique des épidémies. Accompagner ces soldats français de leurs derniers instants à l'inhumation de leurs dépouilles permet de rendre compte de l'expérience collective et individuelle que représente la mort dans un corps expéditionnaire projeté loin de ses bases. Et peut-être de réhabiliter le souvenir de ces hommes en cherchant à nuancer l'assertion de l'immense Homère pour qui "du combat, seuls les lâches s'écartent".

\footnotetext{
${ }^{1}$ Chenu J.-C., 1865, Rapport au conseil de santé des armées sur les résultats du service médico-chirurgical aux ambulances de Crimée et aux hôpitaux militaires français de Turquie, pendant la campagne d'Orient en 18541856-1856, , p. 579.

${ }^{2}$ Afin d'éviter de surcharger l'appareil critique, toutes les citations issues du carton G 259 ne reverront pas à des notes de fin.

${ }^{3}$ Chenu J.-C., op.cit., p. 579.

${ }^{4}$ Fay C.-A., 1889, Souvenirs de la guerre de Crimée, 1854-1856, p. 26.

${ }^{5}$ Bazancourt C., 1857, L'expédition de Crimée jusqu'à la prise de Sébastopol : chroniques de la guerre, p. 98.

${ }^{6}$ Damas A., 1857, Souvenirs religieux et militaires de la Crimée, p. 11.

${ }^{7}$ Cuvillier A., 1860, Le Capitaine Pruvost, quelques traits de sa vie, souvenirs de la guerre de Crimée, Lille, impr. de Lefort, p. 120.

${ }^{8}$ Ibidem. p. 120

${ }^{9}$ Chenu J.-C., op.cit, p. 11.

${ }^{10}$ Scrive p. 36

${ }^{11}$ Chenu J.-C., op.cit p.73

${ }^{12}$ Conseil de santé des armées, Précautions à prendre pour conserver la santé des troupes en orient, 13 mai 1854 , Service Historique de la Défense - Vincennes, série G (Crimée), carton 2.

${ }^{13}$ Général Canrobert, Ordre général n¹60, Sébastopol 4 janvier 1855 , Service Historique de la Défense Vincennes, série G (Crimée), carton 119.

${ }^{14}$ Chenu J.-C., op.cit., p. 119 . .

15 Maréchal Saint-Arnaud, Ordre général n 92, Varna 9 aout 1854, Service Historique de la Défense - Vincennes, série G (Crimée), carton 119

${ }^{16}$ Marroin A., 1861, Histoire médicale de la flotte française dans la mer Noire pendant la guerre de Crimée, $\mathrm{p}$. 116.

17 Général Canrobert, Ordre général n 160 , Sébastopol 4 janvier 1855, Service Historique de la Défense Vincennes, série G (Crimée), carton 119.

${ }^{18}$ Chenu J.-C., op.cit., p 664.

${ }^{19}$ Chenu J.-C., op.cit., p 664.
} 


\section{Bibliographie}

Arzalier J.-J ., 2010, «Les services de santé face à la guerre de Crimée (1854-1856). Etude comparative de laPrise en charge sanitaire des armées britanniques et françaises en Orient » dans Belmas E.\& Nonnis-Vigilante S. (éds), La santé des populations civiles et militaires : nouvelles approches et nouvelles sources hospitalières, XVIIe-XVIIIe siècles, Villeneuve d'Ascq, Presses universitaires duSeptentrion, pp. 151-175.

Chesnais J.-C., 1976, « Les morts violentes en France depuis 1826. Comparaisons internationales »dans Population, n³, 1976. pp. 649-653.

Curtin P.-D., 1989, Death by Migration. Europe's Encounter with the Tropical World in the Nineteenth Century, New York, Cambridge University Press.

Figes O, 2012, The Crimean War. A History, New York, Picador.

Fredj C., 2010, «Compter les morts de Crimée : un tournant sur l'identité professionnelle des médecins de l'armée française (1865-1882) »dans Histoire, économie \& société, 3, pp. 95108.

Gabriel R.-A., 2013, Between Flesh and Steel: A History of Military Medicine from the Middle Ages to the War in Afghanistan, Washington D.C., Ptomac Books.

Gouttman A., 2003, La guerre de Crimée 1853-1856 : la première guerre moderne, Paris, Perrin.

Holden Reid B., 2001, Atlas des guerres - L'âge industriel 1854-1871, Paris, Autrement.

Houdaille J.,1977, « La mortalité (hors combat) des militaires français à la fin du XVIIIe et au début du XIXe siècle » dans Population, n ${ }^{\circ}$, pp. 481-497.

Lemaire M., 2006, La guerre de Crimée : chronique et analyse d'un désastre sanitaire (18541856), thèse de doctorat réalisé sous la direction du Professeur Jean-Charles Jauffret, Université Montpellier

Meslé F. \& Vallin J., 1989, «Reconstitution de tables annuelles de mortalité pour la France au XIXe siècle »dans Population, ${ }^{\circ}$ 6, pp. 1121-1158.

Smallman-Raynor M. R. and Cliff, A. D., 2004, War Epidemics: An Historical Geography of Infectious Diseases in Military Conflict and Civil Strife, 1850-2000, Oxford, Oxford University Press.

Trimaille G., 2010, «L'expertise médico-légale face aux perversions : instrument ou argument de la justice? », dans Droit et cultures, $n^{\circ} 60$, pp.73-87. 